\title{
Radiology Clinic Management in COVID-19 Pandemic
}

\author{
(D) Recep Yılmaz Bayraktarlı, (D) Suzan Deniz Önol, (D) Kemal Harmancı, (D) Duygu Karahacıoğlu, (D) Hülya Yıldız \\ University of Health Sciences Turkey, Prof. Dr. Cemil Taşçıoğlu City Hospital, Clinic of Radiology, İstanbul, Turkey
}

\section{Abstract}

After the outbreak of a Coronavirus Disease-2019 pneumonia occured in Wuhan, China in December 2019, the disease first reached Iran, Europe and then our country on March 11, 2020, creating a pandemic. Our hospital and radiology clinic aimed to overcome the pandemic with minimum damage by taking the necessary precautions. The radiology clinic led treatment management with computed tomography which was stated to be of high diagnostic value. In this informative article, the studies carried out in the radiology clinic during the pandemic were conveyed for information and guidance.

Keywords: Coronovirus, COVID-19, pandemic, tomography, management

\section{INTRODUCTION}

A new Coronavirus Disease-2019 (COVID-19) pneumonia emerged in Wuhan, China, in December 2019.

In this new disease, severe pneumonia symptoms with fever, fatigue, dry cough and respiratory distress were observed. A new corona virus named 2019 Novel Coronovirus Severe Acute Respiratory syndrome-coronavirus-2 (SARS-CoV-2) was declared by the World Health Organization (WHO) as the causative agent of pneumonia in the analyzes performed from samples taken from the lower respiratory tract. Since the incubation time of the virus was an average of 5.2 days and the disease did not show symptoms during the incubation period; its spread to the world was rapid, and WHO declared the outbreak as a global emergency on February 5, 2020 (1).

According to the guidelines in the current medical literature, the gold standard in the diagnosis of SARS-CoV-2 infection is the reverse transcriptase polymerase (RT) chain reaction $(P C R)$ test performed in respiratory tract samples. In the first applications, the sensitivity of RT-PCR was found to be between 37 and $71 \%$. Ai et al. (2) stated that the sensitivity of computed tomography (CT) was $97 \%$ and the specificity was $25 \%$ in a study which was performed in 1014 patients in Wuhan, China and of which PCR results were shown as the reference standard. Similar results have been found in several other publications, and the results suggest that in patients with highly suspected COVID-19 pneumonia, CT imaging will be useful in early detection of interstitial pneumonia (3). It is stated that imaging is of great importance in evaluating the severity and progression of the disease in COVID-19 pneumonia (4). Therefore, radiologists should be aware of the imaging signs of the new COVID-19 pneumonia.

Generally, CT imaging findings are quite specific. However, there may be atypical involvement (single focus, single lobe) or non-specific findings. Again, it is not possible to distinguish COVID-19 pneumonia from other viral pneumonia caused by H1N1 influenza or cytomegalovirus or from atypical pneumonia in terms of CT findings. If the patient has a history of contact and the clinical course is compatible, the diagnosis is made by considering it in favor of COVID-19 pneumonia and by confirming with PCR test. Since the PCR test kits are not diagnostic at the desired level, it is of great importance to perform thoracic CT rapidly and interpret it. 


\section{Radiology Clinic During the Pandemic}

Approximately 1.5 months after the first patients in China, on February 19, 2020, the first patient with COVID-19 was reported in the city of Kum, Iran. Due to the fact that the city of Kum is a religious center and has many visitors around the world, it has been thought that this will have high effect on the spread of the disease. For this reason, considering that it would be inevitable to spread to our country, the planned seminar was immediately canceled due to the need to renew the knowledge of our residents and specialists in terms of this new disease with the leadership of the education supervisor in our clinic and instead, seminars about the danger of COVID-19 in the world, prevention methods and radiological findings in the literature were organized and awareness was raised among all radiologists working in the groups.

In the thorax CT obtained in the radiology clinic, images compatible with the findings of COVID-19 were observed for the first time on March 9 in a hospital employee, and it was reported to the infectious diseases clinic and taking necessary measures was recommended. (Figure 1). Although the first PCR test was negative for COVID-19, as stated in the literature, it was insisted that the findings could be related with COVID-19, and at the end of 10 days, the repeated PCR test was positive for COVID-19.

In our country, 6 days after the first patient was announced on March 11, patients began to come to our hospital, and CT findings of COVID-19 were observed and reported in the thoracic CT examination of 7 patients performed on March

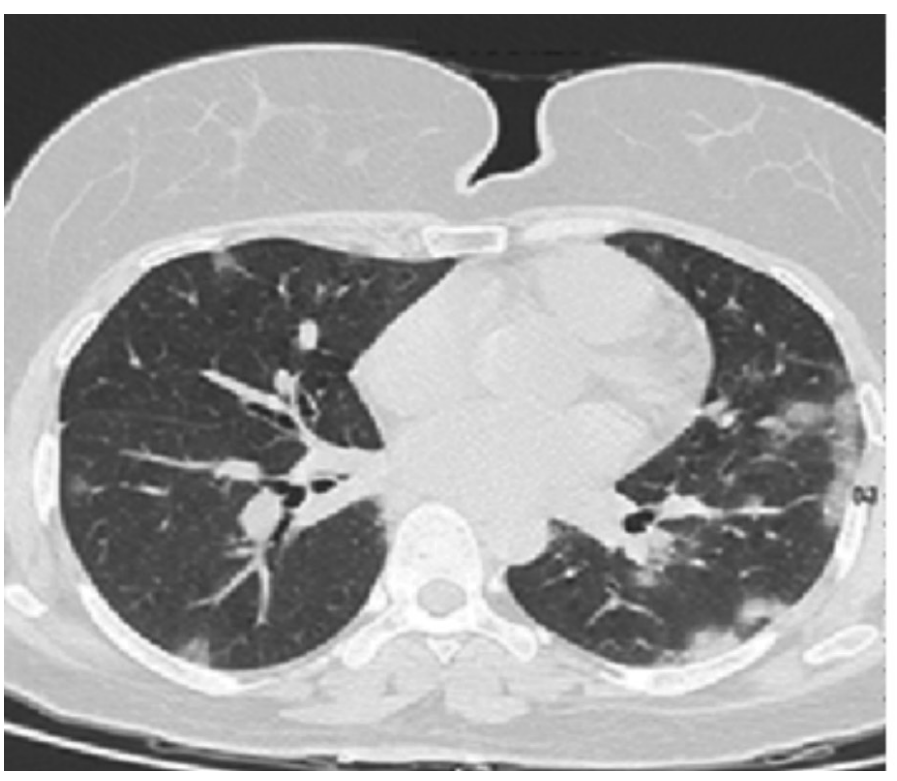

17, the first day. Considering that the disease would spread due to the pandemic, it was decided that we, as the team reading thoracic CT scans, should assit the clinicians in terms of rapid diagnosis and treatment guidance. It was deemed a duty to help clinicians in this triage by realizing the difficulty of how to guide patients in this new unknown situation. For this purpose, in order to serve up CTs, which have a significant role in the differential diagnosis of patients, to clinicians a thoracic CT subgroup for the pandemic was established in the radiology clinic and started reading 24/7 thoracic CTs on March 17. In order to be effective, an instant messaging group was created and it was named "Thorax CT-Vigilance Group". Radiologists, emergency physicians and infectious diseases specialists were added to the group at the first stage. Thus, interactive communication was provided between the emergency service, internal departments and radiology. The first admission examination, clinical findings and thoracic $\mathrm{CT}$ of the patients were evaluated quickly. Thus, the patient's CT scan was immediately evaluated by the radiologist from the system. The relevant radiologist was able to report his/her first finding to the group on his/her mobile phone and sent it to the clinician within half an hour.

Stating that this task will be difficult with only the thorax team due to the increase in the number of patients, our clinical management planned to form a strong union with all radiologists working in the clinic to participate in this important task. Subbranches of radiologists such as "abdomen", "neurology" and "musculoskeletal" were temporarily suspended, and all

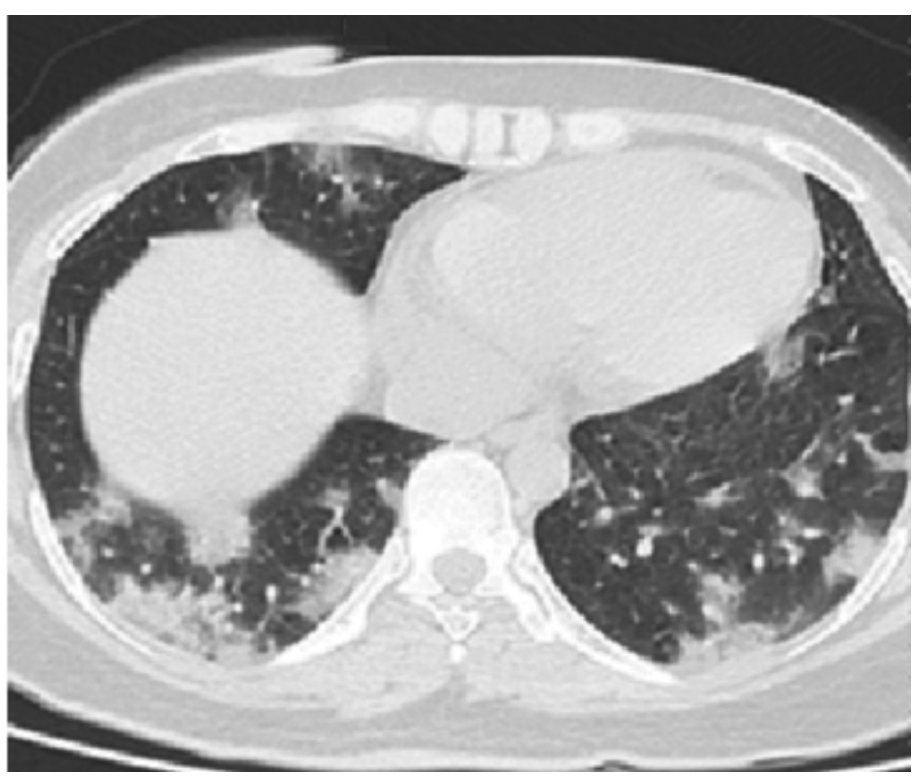

Figure 1. The imaging of the first patient diagnosed as having COVID-19; a 45-year-old female patient, findings consistent with specific viral pneumonia in both lungs, mostly peripheral and subpleural localized, irregularly demarcated multiple consolidation and ground glass opacity areas COVID-19: Coronavirus Disease-2019 
radiologists and residents took the task of reading and reporting thoracic CTs in a very short time and providing guidance.

In our clinic, 7 radiologists, 2 at night and 5 during the day, actively evaluated and reported on thoracic CTs in three shifts over 8 hours. In addition, on-call duties were created for ultrasonography, magnetic resonance imaging, and interventional radiological procedures in the clinic. In hospitals that did not read and report CTs on-site and only received services, such as our hospital, the difficulty in setting up a team in emergency situations and communicating with the consultation team, and the difficulties that clinicians had because of staying away from radiologists who were their deep eyes, were observed. During this pandemic, which was not experienced before and was rare, the handicaps and difficulties of remote radiological reading and reporting were also clearly noticed.

Due to the growth of the group reading thoracic CTs, the reading method and performing criteria were updated (Table 1).

Radiology clinic workers are in the group of healthcare professionals who will be affected first in the pandemic since they encounter patients who have made the first admission and have not been diagnosed (4). Therefore, it is important to protect employees. At the beginning of the pandemic process, all employees were trained by the infectious diseases clinic about general information about COVID-19, ways of transmission and ways of protection, and which personal protectors to use. Again, the working conditions were arranged taking into account the declarations of the Turkish Radiology Association. Our technicians who greeted the patient protected themselves with overalls, masks, gloves, goggles or visors while performing CT scan. The environment of CT device, on the other hand, was ventilated as much as possible, and by cleaning the surface by cleaning staff trained for this pandemic provided by the hospital administration department for each CT device, it was tried to prevent contamination from the environment. At the end of this process, only one of our young $\mathrm{CT}$ technicians had the disease.

Our thorax CT protocols were performed in accordance with the guidelines with a standard dose at first and low dose protocol was not applied. Thin section axial plan imagings with $120 \mathrm{kV}$ and 200-250 mAs and without using intravenous contrast agent were performed. In our hospital, according to the guidelines, very few pediatric patients received CT suitable for automatic child doses (5).

In the following days of the pandemic, in accordance with the guidelines published by the Ministry of Health and with the knowledge of the hospital's scientific committee, low-dose protocol was started to be performed in patients under 20 years of age in the first scan and even in the control scans (COVID-19 guide-12.04.2020) (6).

Interpretation of 3700 CTs with specific viral pneumonia findings with the evaluation of 21,000 thorax CTs performed in our hospital between 17 March 2020-17 May 2020 with 3 different CT devices (Philips Ingenuity core 128 sections, Philips Brillance 40 sections and Toshiba Alexion 16 sections), were reported to clinicians to contribute to their diagnosis. While the findings compatible with specific viral pneumonia were few in the early days, it peaked in mid-April 2020 and reached up to 152 CTs per day and gradually decreased to 10 new patients a day at the end of April. Meanwhile, on the days of curfew, sharp declines were observed in both the number of CTs and the number of those with findings (Chart 1). Another CT device (Toshiba Aquillion LB 16 slice), also available in the radiation oncology clinic in our hospital, was kept ready and clean for possible chaos. Also in our hospital, another tomography device (Philips Brillance Big Bore) serves for radiotherapy applications.

\section{Table 1. Thoracic computed tomography reporting principles of radiologists in pandemic duties}

Our principles of assessing emergency thoracic computed tomographies during COVID-19 pandemic

1- Since early ground glass opacifications may be missed in laptops or normal PC monitors, CTs should be read from medical monitors in workstations in the hospital

2- The evaluating radiologist should photograph the patient's name and the part of the CT scan date and send it to the Thorax CT-vigilance group, and write briefly whether there is findings of viral pneumonia or write information briefly with his/her own sentence

3- If there is a finding in the evaluated CT, "viral pneumonia (+)" should be written briefly in the radiology interpretation section of PACS

4- CT reports should be dictated as quickly as possible (we have a $24 / 7$ reporter) and written reports should be approved quickly (half an hour on average)

5- After the delivery of the duty to the next colleague, the monitor should be left unattended

6- If the radiologist's opinion is requested, the opinion of the radiologist should be reported in other urgent nighttime examinations during the period of duty. CTs that are not asked for opinion will be read by the radiologist who will arrive in the morning

7- In this period, thoracic CTs performed in another hospital and uploaded to the system should also be reported

COVID-19: Coronavirus Disease-2019, CT: Computed tomography, PACS: Picture archiving and communication system 


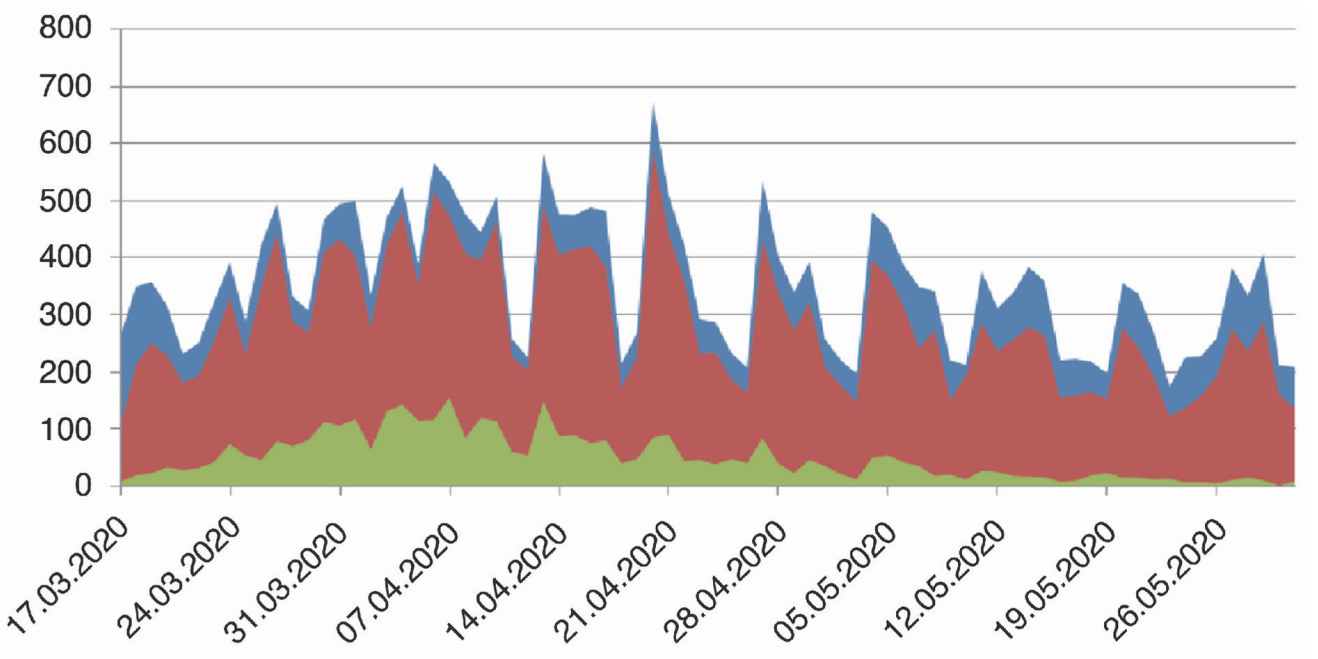

- Number of all CTs

- Number of thorax CTs

n Number of thorax CTs with specific findings of viral pneumonia

Chart 1. The distribution of thorax CTs with specific viral pneumonia findings within the all tomographies over a 43-day period. The sharp declines on the days of the curfew are evident in the chart

CT: Computed tomography

\section{CONCLUSION}

The COVID-19 pandemic that shooks the world is also very effective in our country and has deeply shaken our daily lives. With the fast, effective and sharing approach of our health system, the deadly effects of the pandemic have been slightly overcome and the pandemic has been taken under control.

Our hospital and radiology clinic have played a very effective role in the fight against the pandemic with their pioneering behavior towards the pandemic and their applications in parallel with the practices of our country. In this process, the radiology clinic has coordinated quickly with emergency clinic and infectious diseases clinic with a multidisciplinary approach and has made a great contribution to the triage and treatment of patients. The importance of the effectiveness of communication between clinics has been observed clearly in this process.

In hospitals that did not read and report CTs on-site and only received procurement (teleradiology) services and reportings of cross-sectional images such as our hospital, the difficulty in setting up a team in emergency situations and communicating with the consultation team, and the difficulties that clinicians had because of staying away from radiologists who were their deep eyes, were observed.
Radiologists should work with clinicians as part of a team while helping clinicians by showing them the invisible side of the iceberg. Administrative and academic arrangements should be made for this.

\section{Acknowledgements}

We would like to thank our radiologist colleagues who have made great efforts and contributions in the effective and active role of the radiology clinic during the pandemic process; Hakan Önder, Aslı Ertürk, Deniz Alagöz, Lerzan Çelebi, Yüksel Demir, Deniz Special, Birsen Cihan, Belma Aslan, Naciye Kış, Defne Gürbüz, Serkan Arıbal, Özge Yaper Uğurlar, Tanju Kisbet, Sevil Altunrende, Eyüp Kaya, to Kadir Atakır, to our radiology residents Okan Ince, Melis Koșer, Melisa Yalçın, Burçin Önder, Hamdullah Erk, Eren Özkan, who have greatly supported thoracic CT reading and reporting, to our radiology residents Sinem Aydemir, Remzi Yiğitcan Noyan, Gökhan Aytekin, Sertaç Tatar, who have led the informative and guidance duty of the clinic, to chief technicians Akgül Bulak and Ercüment Tari on behalf of the tomography technicians who have carried tens of thousands of radiological scans, to coordinator Ulaş Altun, to Hüseyin Barutçu on behalf of our secretaries who have ensured the execution of the transactions, and to Hacer Hacı Hanım, Aygül Kelleci and Kazım Keçeci, who have protected us from the disease by purifying every area of the clinic. 


\section{Ethics}

Peer-review: Externally and internally peer-reviewed.

\section{Authorship Contributions}

Concept: R.Y.B., S.D.Ö., D.K., H.Y., Design: R.Y.B., K.H., H.Y., Data Collection or Processing: R.Y.B., Analysis or Interpretation: R.Y.B., S.D.Ö., K.H., D.K., Literature Search: R.Y.B., D.K., Writing: R.Y.B., K.H., H.Y.

Conflict of Interest: No conflict of interest was declared by the authors.

Financial Disclosure: The authors declared that this study received no financial support.

\section{REFERENCES}

1. Li Q, Guan X, Wu P, Wang X, Zhou L, Tong Y, et al. Early transmission dynamics in Wuhan, China, of novel coronavirus-infected pneumonia. N Engl J Med 2020;382:1199-207.
2. Ai T, Yang Z, Hou H, Zhan C, Chen C, Lv W, et al. Correlation of Chest CT and RTPCR Testing in Coronavirus Disease 2019 (COVID-19) in China: A Report of 1014 Cases. Radiology doi: 10.1148/radiol.2020200642.

3. Zu ZY, Jiang MD, Xu PP, Chen W, Ni QQ, Lu GM, et al. Coronavirus disease 2019 (COVID-19): a perspective from China. Radiology doi: 10.1148/ radiol.2020200490

4. Kooraki S, Hosseiny M, Myers L, Gholamrezanezhad A. Coronavirus (COVID-19) Outbreak: What the Department of Radiology Should Know. J Am Coll Radiol 2020;17:447-51.

5. Rubin GD, Ryerson CJ, Haramati LB, Sverzellati N, Kanne JP, Raoof S, et al. The Role of Chest Imaging in Patient Management during the COVID-19 Pandemic: A Multinational Consensus Statement from the Fleischner Society. Radiology doi: org/10.1148/radiol.2020201365.

6. Kang Z, Li X, Zhou S. Recommendation of low-dose CT in the detection and management of COVID-2019. Eur Radiol 2020;30:4356-7. 